\title{
Response Analysis Model of Social Networks using Fuzzy Sets and System Dynamics
}

\author{
Minho Cho \\ Department of Computer System Engineering, Jungwon University \\ 85, Munmu-ro, Goesan-eup, Goesan-gun, Chungbuk, South Korea \\ chominhokr@jwu.ac.kr
}

\begin{abstract}
Research on social network is an important topic in social science. A social network is a realistic environment with both network and interconnection characteristics. This study presents a simulation model of how each node responds to specific inputs (ex. Incentives) to the social networks. In addition, when the setting value of a specific node is changed while simulation is performed, the reaction of the related nodes can be checked in real time. The simulation model is made by considering various environments such as unidirectional, fuzzy set, weighting, and cyclic feedback, so this model can be applied in a complex environment of practical work. Finally, since the simulation model needs to analyze the node response to repetitive input and the relation of each node, we implemented it using Vensim rather than NetLogo.
\end{abstract}

Keywords: Social Science, System Dynamic, Social Network, Simulation, feedback

\section{Introduction}

Simulation of social science is conducted for the purpose of prediction and understanding of a given environment. Figure 1 shows the history of various modeling methods of social science [1].

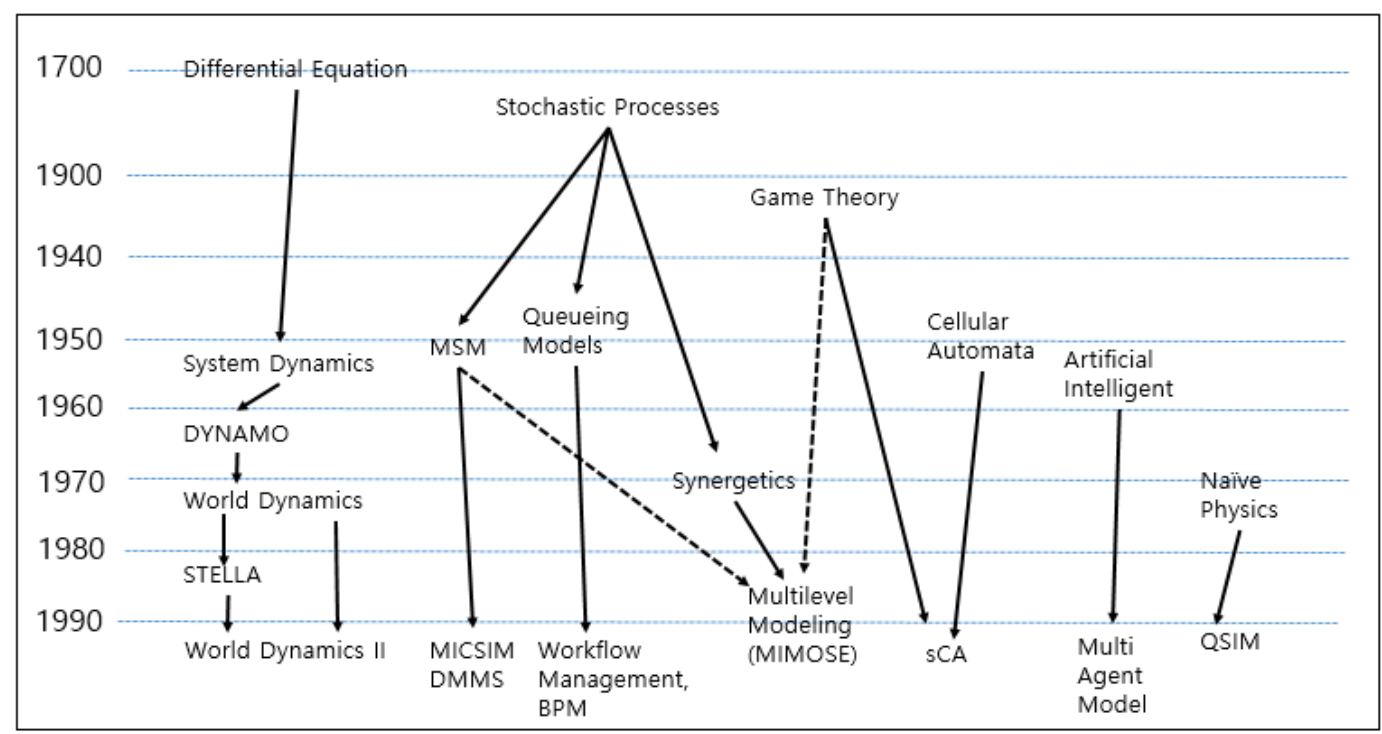

Figure 1. Historical Development of Contemporary Approaches to Social Science Simulation

Received (June 15, 2017), Review Result (September 2, 2017), Accepted (September 8, 2017) 
In recent years, social science simulation has been carried out for social reality, and it has been developing in a way to focus on structure and network by way of social interaction [2]. Based on this, this paper constructs a simulation model for a social network environment as a typical case of Social Science. In particular, social networks have a structure in which member's decision affect each other based on the connections between members [3]. We will identify these features and find ways to use them in practice.

We will check and trace the status change of each node when we provide specific input such as incentives to a configured simulation model. Additionally, we assign weights to the nodes and change them in the middle of the simulation, then we will check at the response of other nodes in real time. This allows you to identify critical nodes, and identify those nodes that have a significant impact on other nodes. Therefore, it will be helpful for practitioners who want to use social networks for business.

\section{Related Research}

\subsection{Social Science Simulation Techniques}

As a method of constructing the social science simulation model, the method using the system dynamics modeling and using the agent based modeling are mainly used [4]. In recent years, the 2D and 3D simulation model using NetLogo have been widely used because of visualization, ease of use and convenience of open source products [5]

The purpose of this paper is to understand the response of each node connected to a particular input. and when the weights for specific nodes are changed during the simulation, we identify the positive and negative responses of the other connected nodes. Therefore, we use Vensim that implements System Dynamics concept rather than NetLogo [6]. By using Vensim, we can easily see the interactions between nodes by feedback concept. For reference, system dynamics is a combination of system and dynamics [7], which means that we are interested in changes over time based on feedback. Vensim, iThink, PowerSim, etc. are used for modeling of system dynamics. In this paper, we will use Vensim for modeling, performing, and analyzing of simulation.

\subsection{Composition of Fuzzy Relation}

There are many ways to express the degree of influence between nodes in a social network. In this study, the degree of influence of each node and link is expressed numerically. However, the relationships between nodes or links are difficult to express in simple number. Therefore, the concept of Fuzzy set is partially introduced to show the association between nodes.

In order to define the fuzzy relation between the two nodes, it is necessary to synthesize the fuzzy relation, which is an extension of the binary relation. When two fuzzy relations $R$ and $S$ are $R \subseteq X \times Y$ and $S \subseteq Y \times Z$, the relation between set $X$ and $\mathrm{Z}$ can be obtained by combining $\mathrm{R}$ and $\mathrm{S}$. Zadeh proposed Max-* synthesis for the synthesis of fuzzy relations, and there are three syntheses of Max-Min, Max-Product and Max-Average [8]. See Figure 2 for details.

Fuzzy composition is mainly used to solve optimization problems. In particular, we use it to solve the linear or nonlinear problem using Max-Min synthesis. However, there have been various attempts to solve nonlinear problems using MaxAverage synthesis and genetic algorithms. In this study, Max-Min synthesis was applied because the influence between nodes is close to linear 


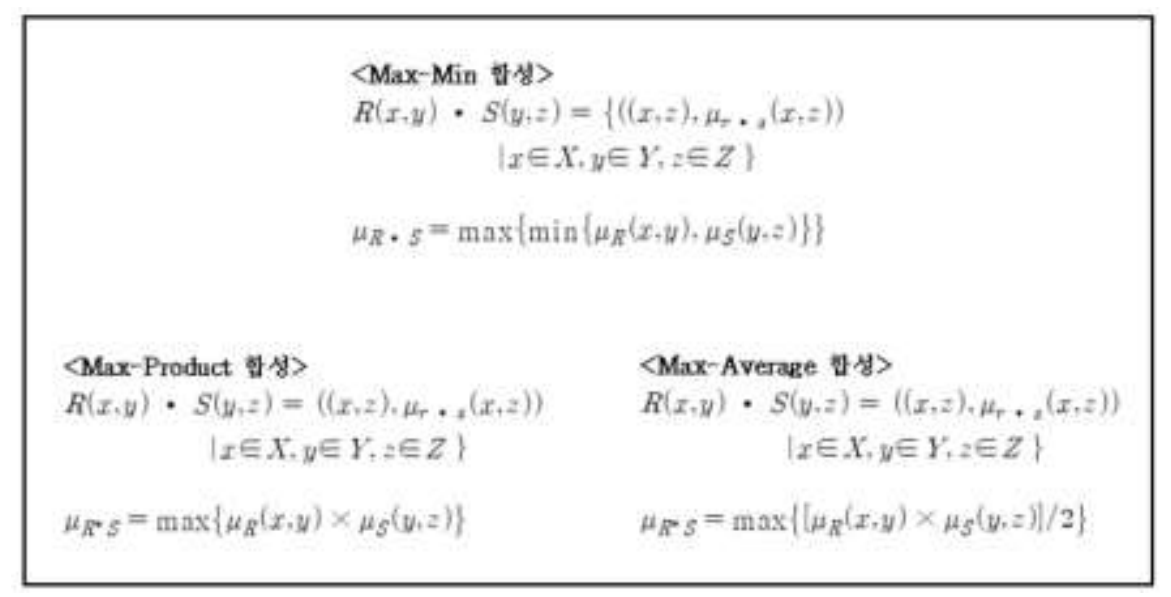

Figure 2. 3 Kind Method of Max-* by Zadeh

\section{Implementation of the Simulation Model}

\subsection{Purpose and Scope}

In this paper, we are creating a simulation model and using it because we want to use social networks for business. Among them, we want to examine the response of each node when we give input like an incentive to social network repeatedly. In addition, we want to examine the effect on the other nodes when the weights assigned to specific nodes are changed (=increasing or decreasing incentives). This is also the process of confirming the word-of-mouth communication used in marketing [9]. To do this, we will construct a small social network with all the characteristics of a real social network, simulate and analyze the response of each node in real time.

\subsection{Summary of Model Implementation Procedures}

The procedure for the simulation modeling is summarized in Figure 3. Simulation modeling is the most important work to be done first. It is then necessary to set the type of data collected for the purpose and the data collected in accordance with the analysis environment. Once this is done, configure the model to suit your needs and set the values of the components. After that, it is necessary to understand whether the result of simulation is consistent with reality. If you do not agree with reality, you need to go back to the previous step and modify or supplement the model. Finally, when the model is completed, perform the simulation and get the results [9].

The most important thing in the process is iterative practice. By iterative practice, we can get the accuracy and reliability result from the model. This is represented by the dotted line in Figure 3. 


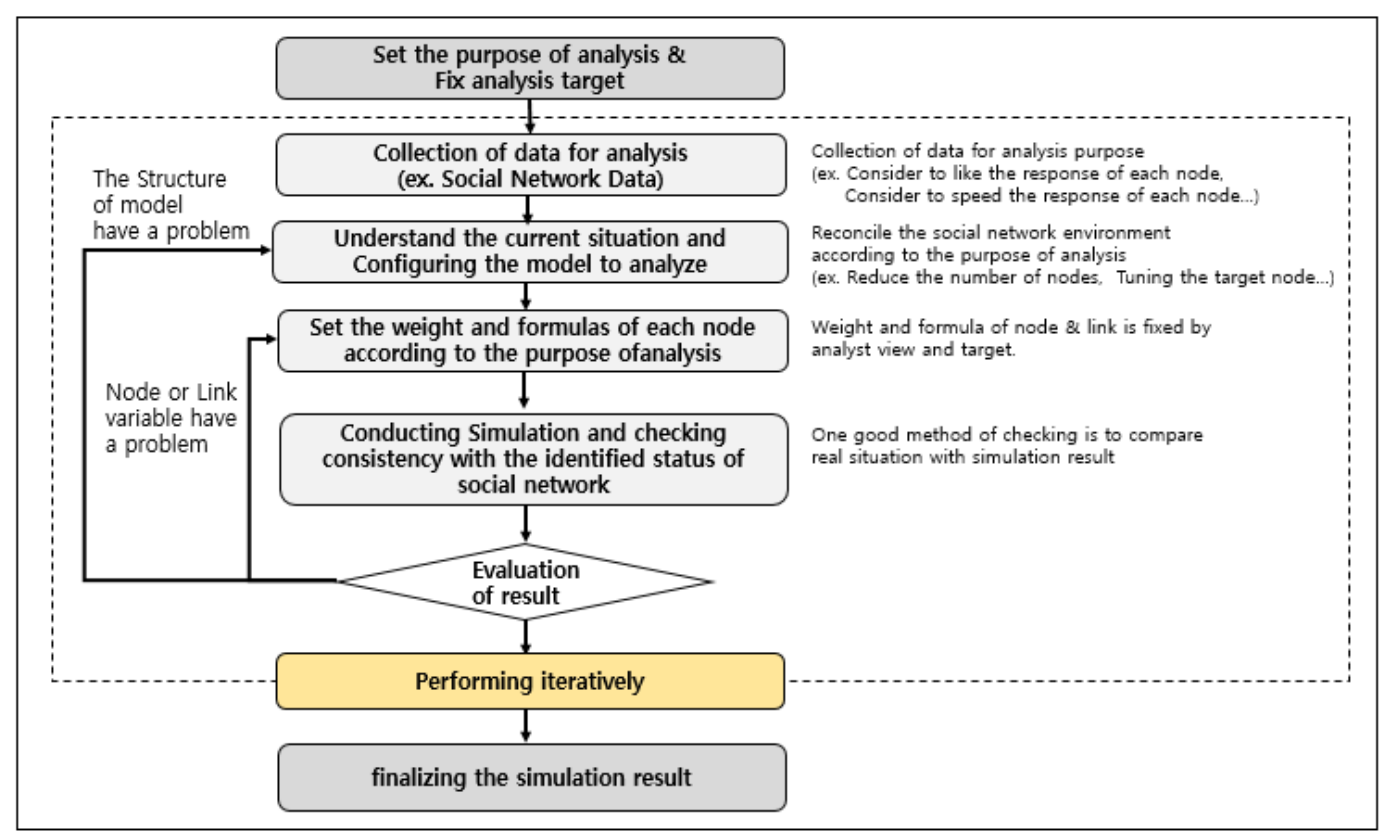

Figure 3. Simulation Modeling Process

\subsection{Construction of Model using Vensim}

The modeling of this study about social network are using Vensim, and it also use Vensim's four kind variables. If you want to use more variable types in case you want to apply in practice, you can refer to the Vensim manual.

Among the various variables provided by Vensim, variables used for the model of this study are Auxiliary, Constant, Level, Initial. In more detail, Auxiliary is used for Link, Constant is used for Initial value \& Node weight, Level is used for Node, Initial is used for Initial value of Node, Other type is not used in this model.

The use of variables provided by Vensim for modeling of this study is summarized as follows.

- Nodes are represented by Box Variables

- Link is expressed as Variable

- Model can set initial values of node, and can set number of iterations for simulation

- Each node has multiple links and accepts indirect feedback.

- The characteristics of each node can be expressed as numerical values, functions, and fuzzy sets

- Each node or link can be weighted separately

The characteristics of this research model are summarized as follows.

- The model consists of links and nodes.

- There is a separate logic for each node, so input and output of node can be controlled by logic

- It is configured to weight each node. Therefore, when a weight assigned to a particular node is changed, it will affect other nodes. This allows you to look at the relationship and dependencies between nodes 
- The link is implemented to have a unidirectional link. However, the model was constructed to allow indirect feedback.

- Each link is implemented in two cases.

$\checkmark$ Has its own logic: There is change in the transfer process between nodes

$\checkmark$ Don't have its own logic: There is no change in the transfer process between nodes

- To identify associations among all nodes, it has a function of assigning an initial input and a repetition number to a specific node.

- There are three ways to set up logic for nodes and links.

$\checkmark$ Assign a separate value to each node and link

$\checkmark$ Use the relationship express between nodes and links

$\checkmark \quad$ Use the Fuzzy set to each node and link

\section{Perform Simulation and Analyze Results}

"What do you need to know if you want to use social networks for your business?" This is the subject of this part. To solve this question, we make a model and perform a simulation, and then we want to reveal the following.

- Analyzes whether the correlated nodes exhibit any response (e.g., positive, negative, increase, decrease...) when specific inputs (e.g., incentives, promotions ...) are given to the entire social network. So, if you want to get the best results on your social network, you can figure out how strong, and how many, specific inputs (incentives, promotions) you have to offer

- We want to analyze the response of other nodes when a particular input is given to the whole network and the weights variation for specific node (e.g., reinforcement of incentives, provision of discounts). Through this, it is possible to grasp the interrelationship between a specific node and another node, and to find an optimal situation for each node

In conclusion, we want to know the conditions for obtaining optimal results in a given social network. Finding out what input, what node, and how many times it can get the best results.

\subsection{Construction of Model}

Simulations for social networks can be configured in many forms. However, Figure 4 shows our simulation model that have the structure of links and nodes with weight and initial value assignments, bidirectional links, fixed value, formula and fuzzy set properties. A constructed model is a virtual and small environment for identifying functions according to a given purpose. However, since it reflects various situations that may occur in a real environment, it can be applied in practice with only small modification. 


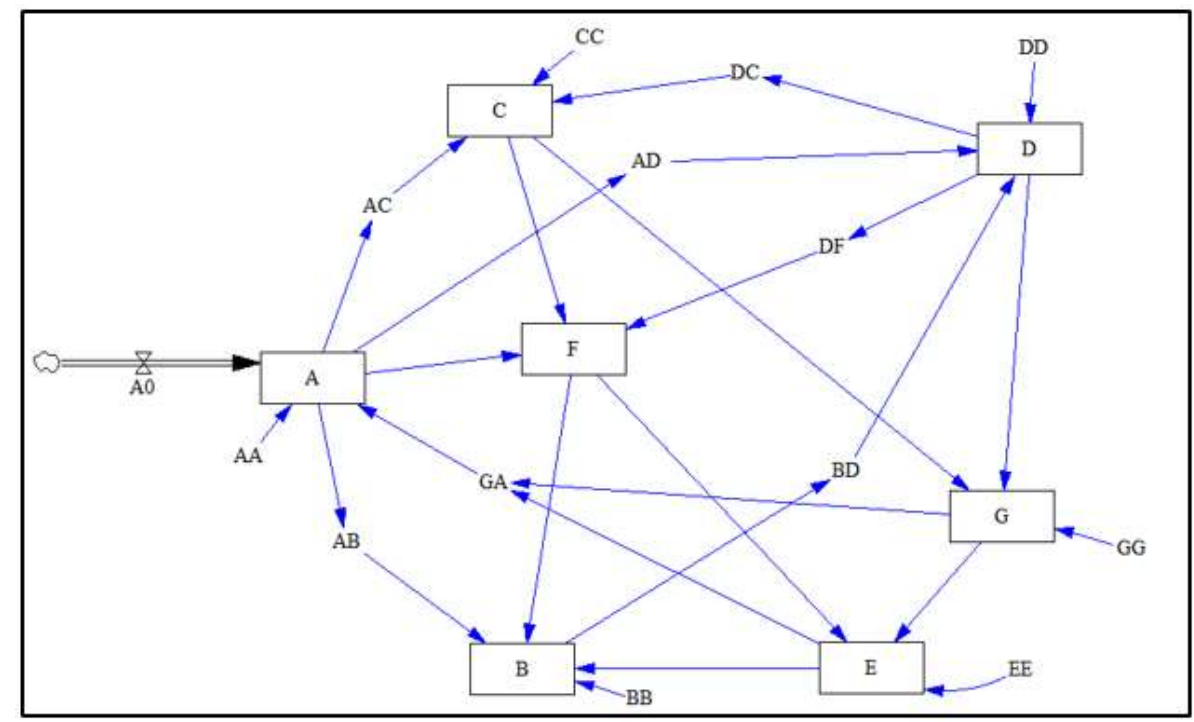

Figure 4. Simulation Model about Social Network

The characteristics of the model provided in Figure 4 are summarized as follows.

- It is a social network consisting of seven nodes

- It has one-way link

$\checkmark \quad$ When a weight is given while being unidirectional

: $\mathrm{AC}, \mathrm{AD}, \mathrm{AB}, \mathrm{BD}, \mathrm{DC}, \mathrm{DF}$

$\checkmark$ When a weight is not given while being unidirectional

: AF, FE, CF, FB, DG, CG, GE

- It has Circular feedback

$\checkmark$ Example: $\mathrm{A}-\mathrm{AC}-\mathrm{C}-\mathrm{G}-\mathrm{GA}-\mathrm{A}$

- F node use Fuzzy set

$\checkmark$ Apply a fuzzy set to the weights of links A, C, DF, B, E and F node

- The fixed weight of the link is expressed by the weight expression of the target node

- It was constructed to change the initial value and the weight per node

The model shown in Figure 4 has the flexibility and extensibility to accommodate various environments in the real world. Through this, you can see what you want to check in the simulation model

\subsection{Variable Setting Information}

Figure 5 shows the information on the nodes and links used in the simulation model presented in Figure 4. 


\begin{tabular}{|c|c|c|c|c|}
\hline $\begin{array}{l}\text { Variable } \\
\text { Name }\end{array}$ & Type & Equation & $\begin{array}{l}\text { Initial } \\
\text { Value }\end{array}$ & Relative Variable \\
\hline A & Level & $\mathrm{A} 0^{*} \mathrm{AA}-\mathrm{GA}$ & 5 & $\mathrm{~A}, \mathrm{AO}, \mathrm{AA}, \mathrm{GA}$ \\
\hline B & Level & $\left(\mathrm{AB}^{*} 0.6-\mathrm{E}^{*} 0.2+\mathrm{F}^{\star} 0.4\right)^{*} \mathrm{BB}$ & 2 & $\mathrm{~B}, \mathrm{AB}, \mathrm{BB}, \mathrm{E}, \mathrm{F}$ \\
\hline C & Auxiliary/Normal & $\left(A C-\left(D C^{*} .7\right)\right)^{*} C C$ & & $A C, C C, D C$ \\
\hline $\mathrm{D}$ & Level & $\left(\left(A D^{*} 0.8\right)-\left(B D^{*} 0.8\right)\right)^{*} \mathrm{DD}$ & 1 & $\mathrm{D}, \mathrm{AD}, \mathrm{BD}, \mathrm{D}$ \\
\hline E & Level & $\left(F^{*} 0.6-G^{*} 0.3\right)^{\star} \mathrm{EE}$ & 1 & $E, E E, F, G$ \\
\hline $\mathrm{F}$ & Level & $\left(A^{\star} 0.8+C^{\star} 0.8-D^{\star} 0.6\right)$ & 3 & $\mathrm{~F}, \mathrm{~A}, \mathrm{C}, \mathrm{DF}$ \\
\hline \multicolumn{5}{|c|}{$\rightarrow F$ is applied to Fuzzy Set } \\
\hline G & Level & $\left(\left(D^{*} 0.3\right)-\left(C^{*} 0.4\right)\right)^{*} G G$ & 1 & G, C, D, GG \\
\hline$A B$ & Auxiliary/Normal & $A^{*} 0.3$ & & A \\
\hline $\mathrm{AC}$ & Auxiliary/Normal & $\mathrm{A}^{*} 0.7$ & & A \\
\hline$A D$ & Auxiliary/Normal & $A^{*} 0.2$ & & A \\
\hline $\mathrm{BD}$ & Auxiliary/with Lookup & $B * 0.2$ & & B \\
\hline DC & Auxiliary/Normal & $D^{*} 0.8$ & & D \\
\hline DF & Auxiliary/Normal & $D^{*} 0.4$ & & $\mathrm{D}$ \\
\hline GA & Auxiliary/Normal & $\left(G^{*} 0.4\right)+\left(E^{*} 0.6\right)$ & & $E, G$ \\
\hline \multicolumn{5}{|c|}{$\mathrm{AA}, \mathrm{BB}, \mathrm{CC}, \mathrm{DD}, \mathrm{EE}, \mathrm{GG} \rightarrow$ Constant/Normal 1} \\
\hline $\mathrm{AO}$ & Constant/Normal & 10 & & \\
\hline
\end{tabular}

Figure 5. Variable List and Equation of Model's Link and Node

The important thing to consider is that the configuration for the node and link are of different types, and the form of the formula that constitutes each node and link are also different because of the situation of node and link is different. We can confirm that the model expresses the relation of each link and node through the formula. The node F constructs the equation by applying the concept of the fuzzy set differently from other nodes. Although we can apply fuzzy sets to other nodes and links, we have applied to only one link and node of simulation model at this stage.

Next, let's make sure we set the weights and initial values for each node. This is a means for expressing the degree of familiarity for each node, and It express its association with other nodes in the model. This can be used to determine how a particular node affects other nodes. In this model, the link only considers the association with the node. Finally, the initial value setting and the number of iterations of simulation (it is not shown in the table) is also an important factor affecting the results generated by the model. The values given in Figure 5 are values that are arbitrarily set to be most similar to the assumed network situation. In practice, it is necessary to set the values of nodes and links to reflect the actual situation. Here, the description of this part is omitted

F node is the node set by the fuzzy set. In this model, the concept of fuzzy set is partially applied. The fuzzy set for the F node should be obtained based on the observed values in the simulation preparation stage. To do this, Prof. Zadeh applied the Max-Min method, which is widely used in linear problems, among the three Max-Min synthesis methods (Max-Min, Max-Product, and Max-Average). See Figure 6 for more information. 


<F's fuzzy set>
\begin{tabular}{|l|l|l|}
\hline & < & E F's new fuzzy set> \\
\hline$A$ & 0.3 & 0.2 \\
\hline C & 0.7 & 0.4 \\
\hline DF & 0.2 & 0.8 \\
\hline
\end{tabular} \mid \begin{tabular}{|l|l|l|}
\hline & $B$ & $E$ \\
\hline$A$ & 0.3 & 0.2 \\
\hline$C$ & 0.7 & 0.4 \\
\hline DF & 0.2 & 0.6 \\
\hline Final & $\mathbf{0 . 7}$ & $\mathbf{0 . 4}$ \\
\hline
\end{tabular}

At Simulation time, $A / C / D F$ 's value is checked

$(A, C, D F) \rightarrow(0.4,0.8,0.6)$.then we can get

F's new fuzzy set using by Max-Min method.

\section{Figure 6. New Fuzzy Set Generation using Max-Min Method}

\subsection{Analysis of the Response of the Nodes to Repetition of a Specific Input}

Social networks (such as Facebook and Twitter) operate services including mobile environment. And there is a continuing need to use social networks for marketing now and in the future. In this case, strategies to optimize the effectiveness of specific social networks are important. In other words, how many marketing events, including incentives and discounts, to whom and how strong, is important.

Figure 7 shows the simulation results of the social network shown in Figure 4. It shows a response of nodes in the social network when 10 promotional events were held at a strength of $10(\rightarrow$ the unit of strength is determined by the user).

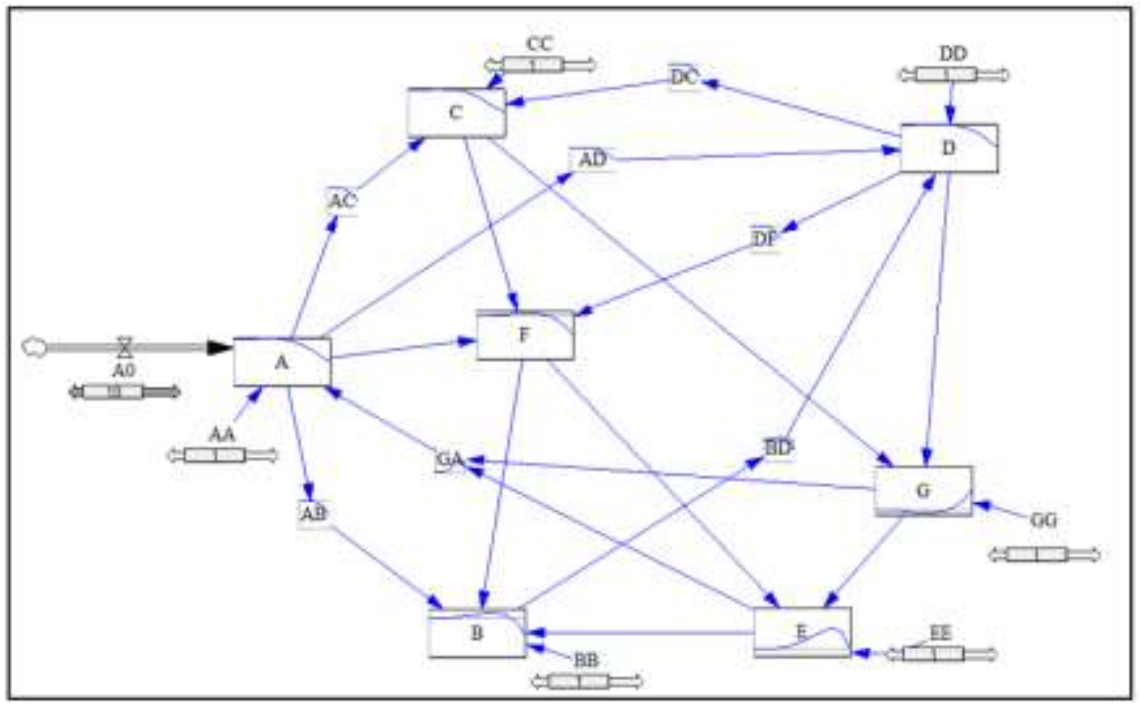

Figure 7. Analysis of Response of Whole Node to Repetition of Specific Input

Result of Figure 7 simulation is as below

- When a specific input is provided to the model, A, C, F, D, and B respond negatively over time

- If a specific input is given to the model, E responds positively and then returns to negative.

- Given a specific input to the model, $G$ reacts positively over time. 
Through simulations, it is possible to check the response of the nodes constituting the network in repeatedly given input. In particular, it is possible to take a separate action on these nodes by finding out the nodes that show negative responses in advance.

In the case of Figure 7, it is noteworthy that the target nodes have a negative response to a particular incentive or stimulus, so what do you need to do to turn them into positive? There are many answers to this, we can be achieved by enhancing support for specific nodes. This is one of the research. In order to simulate the enhancement of support for a specific node, a method of assigning a different weight to a node to be analyzed has been developed. Examples are AA, BB, CC, DD, EE, and GG variables in Figure 7. In Figure 7, we set the value of the related variable to 1 , so that it does not affect the overall simulation. At next step of simulation, we will use it.

\subsection{Analysis of the Response of other Nodes when the Weight of a Specific Node is Changed}

We confirmed the response of all nodes to a specific input. Then, if the problem for the entire node or for the particular node is identified, then we have to think about the appropriate action for that node. For example, you need to see how changing support for a particular node affects other nodes, or to know which node is closely related to that node in the model. In this chapter, we will show how to handle the identified node in our model.

Figure 8 shows an example of the response of other nodes when weights of specific nodes are increased.

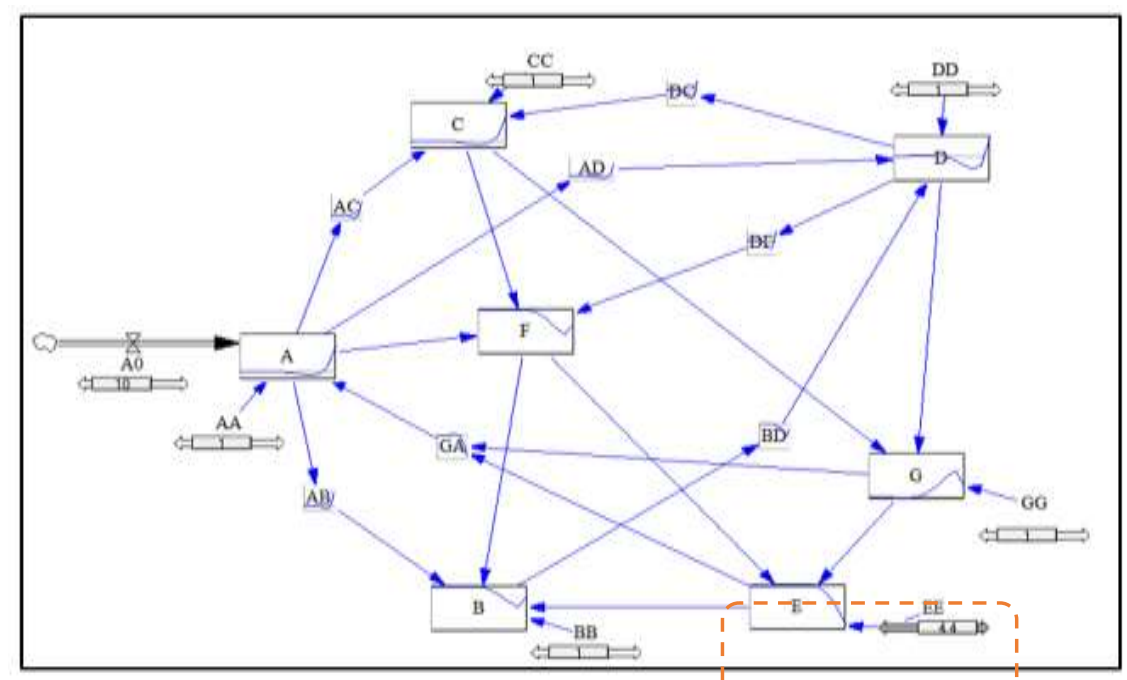

Figure 8. Analysis of Response of other Nodes in Case of the Weight of $E$ Node is Changed

In Figure 7, E node shows a positive response in simulation, so we strengthen support for E node $(1 \rightarrow 4.4)$. Let's summarize the response of each node.

- Node E turns to negative

- Nodes C, F, D, and B are negative and positive

- Node $\mathrm{G}$ is positive and turns to negative.

Finally, as support for node E increases, most nodes will eventually respond positively. However, it is unusual that the response of node $\mathrm{E}$ changes negatively. Anyway, based on these result, we can be seen that the change in weight for $\mathrm{E}$ affects the entire network. 
Figure 9 simulates the effects on other nodes when changing the weights for the $\mathrm{C}$ nodes that showed a negative response to a particular input.

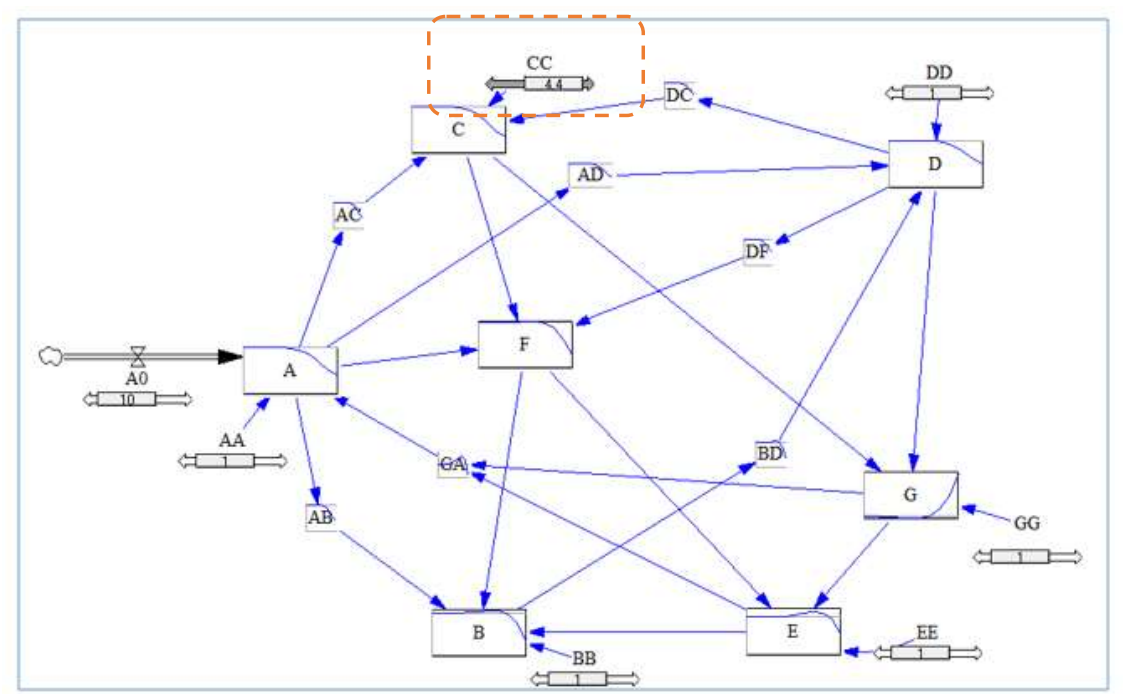

Figure 9. Analysis of Response of other Nodes in Case of the Weight of E Node is Changed

When the weight of node $\mathrm{C}$ is changed $(1 \rightarrow 4.4)$, the responses of other nodes are almost the same. B, F, and D are turning to negative over time. G still shows a positive response. E changes from positive to negative. In other words, additional support for the $\mathrm{C}$ node does not seem to have a significant impact on the entire network. Based on this, we can direct the direction of the marketing strategy for the social network.

For the sake of understanding, the simulation results are summarized as follows:

- For a given input, most of the nodes will react negatively, so providing a separate input (incentive, offering a prize) can be considered meaningless.

- Support for E-nodes (incentives, compensation programs) has a positive effect on all other nodes. However, the E node itself does not like this support

- C nodes have little or no impact on other nodes

It is important for the practitioner to confirm how each node responds to certain inputs to a particular social network. To do this, modeling a social network using Vensim and looking at the response of each node to a given situation is helpful in identifying the characteristics of each node. In addition, providing a separate input to a node or a particular node that exhibits a negative response, and looking at the response of other nodes, provides a way to effectively utilize limited resources to practitioner.

\section{Conclusions}

As the complexity of human social activity, social science is recognized as an important research area. Among them, research on social networks has been conducted in various ways for a long time. In particular, since 2000, social networks such as Facebook and Twitter have become commonplace, there is a growing demand for utilizing this in business execution [10]. In particular, knowing how 
each node responds when specific inputs (incentives, promotions, etc.,) are provided, and when a node is concentrated management in social network, the effect on the entire node is a concern of many practitioners.

In this paper, we are modeling the social network environment on a computer. And we apply feedback concept of system dynamics and fuzzy set to construct environment similar to reality. When the simulation is performed by the modeling method presented in this paper, we can check the response of each node for a given input, and simultaneously check the response of nodes that affect a particular node. This model reflects the directionality of actual social networks, node and link characteristics by using variables and constants, functions, fuzzy sets, and inter node connectivity through feedback.

It is important to note that most of the social networks we are trying to analyze in practice are often large. In such cases, it may be effective to scale down the model that perform the analysis according to the purpose of the analysis (e.g., only for nodes with three or more links). In this case, the concept of the group node [11] can be applied to reduce the size of the social network to be analyzed. If you want to test a simple one-way model, you can refer to the paper published in the Journal of the KIECS [12] and the paper published in the NGCIT 2017[13].

\section{Acknowledgments}

This work was supported by the Jungwon University Research Grants (2017-032).

\section{References}

[1] K. Toitzsch, "Social Science Simulation-Origins, Prospects Purpose", lecture Notes in Economics and Mathematical System, vol. 456, (1997), pp. 41-45.

[2] F. Squazzoni, W. Jager and B. Edmonds, "Social Simulation in the Social Science", Social Science Computer Review, vol. 32, (2014), pp. 279-294.

[3] M. Kakihara, "Understanding real-virtual perspective of human behavior from the active stream perspective", Pacific Asia Conference on Information Systems, Taipai, (2010) July 9-12.

[4] C. Revilla, "Computational Social Science", Willy Interdisciplinary Reviews: Computational Statistics, vol. 2, no. 3, (2010), pp 259-271.

[5] S. Tisue and U. Wilensky, "NetLogo: A Simple Environment for Modeling Complexity", International Conference on Complex System, Boston, USA, (2004) May 16-21.

[6] P. Otto and M. Simon, "Dynamic perspectives on social characteristics and sustainability in online community networks", System Dynamic Review, vol. 24, no. 3, (2008), pp. 321-347.

[7] J. Forrester, "The Beginning of System Dynamics", Banquet Tal at the International meeting of the System Dynamics Society, Stuttgart, German, (1989).

[8] I. Lee and K. Shin, "A Study on Forecasting Accuracy Improvement of Case Based Reasoning Approach Using Fuzzy Relation", Journal of the Intelligence and Information System, vol. 16, no. 4, (2010), pp. 67-84.

[9] M. Truson, R. Buckin and K. Pauwels, "Effects of word-of-mouth versus traditional marketing: Finding from an internet social network site", Journal of Marketing, vol. 73, (2009), pp. 90-102.

[10] S. Hong, "The Effect of Social Network Usage on the Formation of Social Capital: Based on Twitter, Facebook, KakaoStory", J. of the KIECS, vol. 10, no. 7, (2015), pp. 837-843.

[11] M. Cho, "Identification of Group Node using Genetic Algorithm and Re-Construction Technique of Social Network", J. of the KIECS, vol. 10, no. 7, (2015), pp. 837-843.

[12] M. Cho, "Modeling and Simulation of Social Network using Correlation between Node and Node weight”, J. of the KIECS, vol. 10, no. 7, (2015), pp. 837-843.

[13] M. Cho, "Simulation Model of Social Network Environment using System Dynamics", Proceedings of the $6^{\text {th }}$ International Conference on Next Generation Computer and Information Technology, Ho Choi Minh, Vietnam, (2017) August 16-18. 


\section{Author}

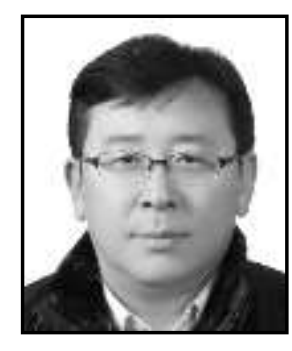

Minho Cho, Author's profile. Minho Cho received the B.S. degree in industrial engineering from the University of InHa, Incheon, South Korea, in 1989, the M.S. degrees in computer engineering from SoongSil University, Seoul, South Korea, in 1998, and the Ph.D. degree in computer engineering from University of SoongSil, Seoul, South Korea, in 2003. He was a senior research engineer from Feb. 1989 to Mar. 2000 in Hewlett-Packard Korea, Seoul, South Korea. $\mathrm{He}$ is currently with the department of Computer System Engineering in Jungwon University, Goesan, South Korea. His research interests are Software Engineering, Social Science, Statistical Analysis. 